\title{
USO DE ALGORITMOS GENETICOS PARA RESOLVER EL MODELO DETERMINISTAY ESTOCASTICO PARA EL DISENO DE UNA RED DE RECOGIDA DE RESIDUOS
}

\author{
MIGUEL ORTEGA-MIER \\ JOAQUIN DELGADO HIPOLITO \\ ALVARO GARCIA-SANCHEZ \\ DEPARTAMENTO DE INGENIERIA DE ORGANIZACION, ADMINISTRACION DE EMPRESASY ESTADISTICA \\ ESCUELA TECNICA SUPERIOR DE INGENIEROS INDUSTRIALES \\ UNIVERSIDAD POLITECNICA DE MADRID
}

\begin{abstract}
Resumen: Unos de los problemas que aparecen en la gestión de las cadenas de suministro inversas es el de las decisiones relativas a la localización de plantas de tratamiento, centros de recogida y de transferencia. En esta comunicación se presentan un modelo determinista MIP y el modelo estocástico derivado para ayudar a la decisión relativa a la localización de una planta de tratamiento y los centros de transferencias necesarios para reducir los costes totales de funcionamiento de un sistema de recogida de residuos. Estos modelos se intentan resolver de forma exacta con CPLEX 10.0 y dada la dificultad se plantea un enfoque distinto utilizando algoritmos genéticos y programación lineal continua.
\end{abstract}

Palabras clave: Recogida residuos, logística inversa, algoritmos genéticos.

\section{Introducción}

Uno de los problemas que aparecen en la gestión de las cadenas de suministro es la localización de las instalaciones clave de dicha cadena (plantas de producción, almacenes, etc.) En el área de la logística inversa este problema de diseño se traduce en las decisiones acerca de la localización de los diferentes centros de recogida, centros de transferencia o plantas de tratamiento.
Existen muchos modelos matemáticos que intentan facilitar la toma de estas decisiones a ese respecto. Cuando las implicaciones de estas decisiones son a largo plazo es muy difícil de estimar, con precisión, la evolución de los valores de todos los parámetros y las variables de los modelos correspondientes.

Esta situación es muy frecuente cuando se construyen modelos de logística inversa. Este es el caso por ejemplo de la evolución de los costes de los combustibles o la cantidad de residuo generado a lo largo de los años.

En el siguiente apartado se presenta un problema de localización de instalaciones de recogida y tratamiento de residuos. En los siguientes apartados se ofrece un modelo MIP determinista que resuelve el problema previamente anunciado y el modelo estocástico asociado. Tras comentar la dificultad asociada a la resolución de dicho modelo para casos de gran tamaño, en el último apartado se presenta una metodología distinta basada en los algoritmos genéticos.

\section{Presentación del problema}

El problema que se presenta, muy habitual cuando se quiere diseñar una red de recogida de residuos (plásticos, RSU, etc.) es el siguiente. Dados una serie de municipios en los que se genera residuo, se trata 
de determinar en qué municipio colocar la planta de tratamiento a la cual enviar dichos residuos y los posibles centros de transferencias donde llevar el residuo desde cada municipio que, una vez compactado, se transportar hasta la planta de tratamiento. El objetivo es incurrir en el menor coste posible durante un horizonte contemplado de varios periodos, pudiendo colocar la planta de tratamientos y los centros de transferencia en cualquier municipio. Se admite que en ese horizonte no tiene sentido cambiar la localización de la planta pero quizás sí el número o la localización de los centros de transferencia.

Este problema surge principalmente cuando la red de recogida no existe y, por lo tanto, se tiene que diseñar el sistema de recogida partiendo de cero.

La recogida del residuo se realiza con unos camiones recolectores que están preparados para recoger el residuo desde los contenedores de la calle y que transportan el residuo sin compactar al centro de transferencia correspondiente. Una vez en el centro de transferencias, el residuo se compacta y, cuando un remolque está lleno, es transferido en trailer a la planta de tratamiento. En este problema, cobra mucha importancia la relación entre los costes de recogida del camión recolector y los costes de transferencia. La relación entre estos costes de transporte y recogida, junto con los costes fijos de los centros de transferencia, llevarán a soluciones en las que se utilizan más o menos centros de transferencia.

En la Figura I (izda.) se muestra el conjunto de municipios en los que se genera residuo. Una posible solución del problema se ofrece en la misma figura (dcha.); en este caso ya se ha localizado la planta de tratamiento (cruz) y se ha utilizan tres centros de transferencia (triángulos).

\section{El modelo de localización de una planta de tratamiento y los centros de transferencia asociados: STPNTCLP (Single Treatment Plant and Necessary Transfer Centers Location Problem)}

Se ha desarrollado un modelo matemático de localización dinámica asociado al problema presentado en el apartado anterior, que se ha llamado «Single Treatment Plant and Necessary Transfer Centers Location Problem» (STPNTCLP).

Por un lado, en la literatura existen numerosos artículos que tratan de localización dinámica (Saldanha, 1998; Antunes, 2000; Hinojosa, 2000; Bose, 2003) y; por otro, sobre modelos de logística inversa (Fleischmann, 200I).

En el modelo STPNTCLP se admiten las siguientes hipótesis:

- El modelo es dinámico. Se contempla un horizonte dividido en varios periodos (para este problema, años).

- La localización de la planta de tratamiento es fija para todo el horizonte contemplado. El coste fijo de la planta de tratamiento se supone independiente de dónde se localiza.

- Los centros de transferencia se pueden cambiar cada periodo si eso permite reducir los costes totales de la gestión. Tienen un coste fijo por periodo asociado por su uso. Los costes de apertura y cierre no son relevantes.

- No se tienen en cuenta costes de recogida del residuo dentro de cada municipio, se admite que no tienen relevancia ya que pueden ser los mismos

Figura I

Situación inicial del problema. La planta de tratamiento y los centros están por elegir (izda.). Solución del problema. La planta de tratamiento y los centros de transferencia están elegidos (dcha.)

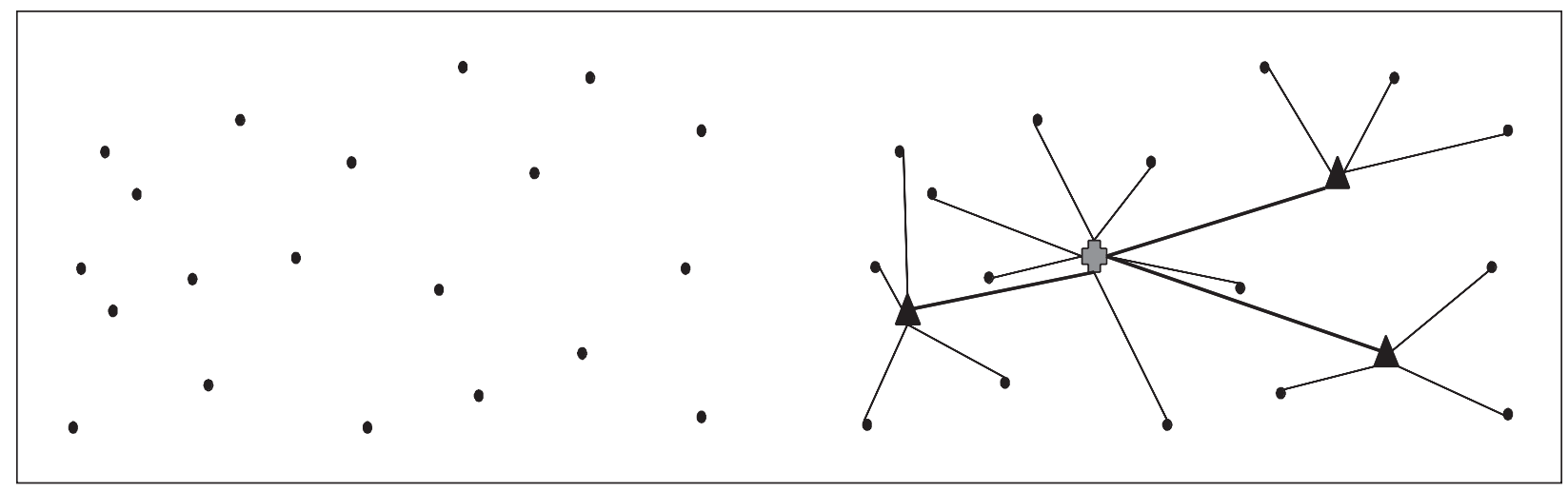


independiente de la decisión que se tome. Esta hipótesis es aceptable cuando los municipios son urbanos, y no tanto cuando un municipio esté compuesto por poblaciones o barrios dispersos.

- Los costes de transporte del residuo y los de transferencia son proporcionales a las distancias existentes entre el lugar de origen y destino de los mismos. Esto supone que no se tienen en cuenta rutas, lo cual se puede admitir, dado que este problema se considera estratégico. Una vez decididas las localizaciones sí se pueden calcular las rutas correspondientes.

En las siguientes tablas, se puede observar cuáles son los índices, parámetros y variables utilizados en el modelo.

En la Figura 2 se refleja claramente el significado de cada una de las variables utilizadas en el modelo.

El modelo matemático correspondiente al STPNTCLP se expresa de la siguiente forma:

$\min \sum_{j, t} F \cdot y_{j t}+\sum_{j, k, t} h_{j k t} \cdot d_{j k} \cdot c T R+\sum_{i, j, t} x_{i j t} \cdot d_{i j} \cdot c R E$

Tabla I

Índices del problema STPNTCLP

\begin{tabular}{|c|l|c|}
\hline Índices & \multicolumn{1}{|c|}{ Descripción } & Rango \\
\hline$i$ & Municipios productores de residuo & I ..M \\
\hline$j$ & Posibles centros de transferencia & I ..M \\
\hline$k$ & $\begin{array}{l}\text { Municipios candidatos para la } \\
\text { planta de tratamiento }\end{array}$ & I ..M \\
\hline$t$ & Períodos & I..T \\
\hline
\end{tabular}

Figura 2

Significado de las variables del modelo

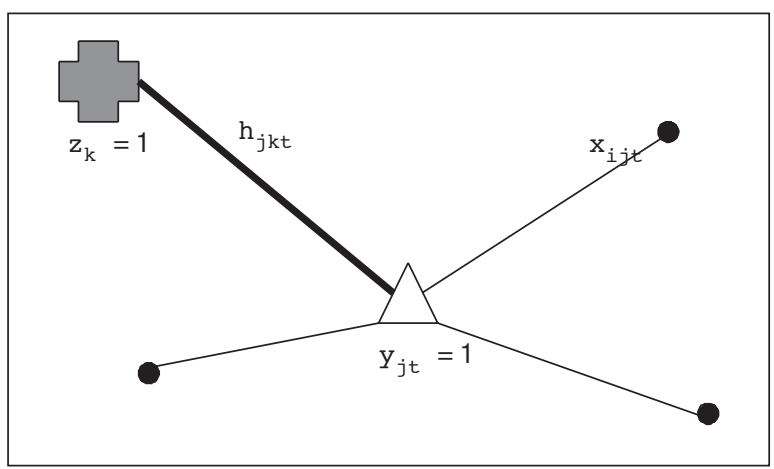

sujeto a:

$\sum_{j} x_{i j t}=A_{i t}, \forall i, \forall t$

$x_{i j t} \leq y_{j t} * C A P, \quad \forall i, \forall j, \forall t$

$\sum_{i, j} x_{i j t} \leq \sum_{j} y_{j t} * C A P, \quad \forall t$

$\sum_{k} h_{j k t}=\sum_{i} x_{i j t}, \forall j, \forall t$

$\sum_{j} h_{j k t} \leq z_{k} *$ CAPplanta, $\forall k, \forall t$

$\sum_{k} z_{k}=1$

\section{I. Solución exacta}

Este problema es NP-HARD (Garey, 1979) y resulta difícil resolverlo de forma exacta. Se han intentado resolver varios casos de complejidad creciente

Tabla 2

Parámetros del problema STPNTCLP

\begin{tabular}{|c|c|}
\hline Parámetros & \multicolumn{1}{c|}{ Descripción } \\
\hline$A_{i t}$ & Producción de residuo de cada municipio en el año $t$ (en Tm) \\
\hline$C A P_{j}$ & Capacidad anual de un centro de transferencia $j$ (en Tm) \\
\hline$c T R$ & $\begin{array}{l}\text { Coste medio de transferencia del residuo compactado entre un centro de transferencia y la planta de } \\
\text { tratamiento (en } € / \mathrm{km} \cdot T \mathrm{~m})\end{array}$ \\
\hline$C R E$ & $\begin{array}{l}\text { Coste medio de transporte del residuo recogido en cada municipio y llevado al centro de transferencia } \\
\text { (en } € / \mathrm{km} \cdot T \mathrm{~m})\end{array}$ \\
\hline$F_{j}$ & Costes fijos del centro de transferencia j por período (en $€$ ) \\
\hline
\end{tabular}


Tabla 3

Definición de las variables del problema STPNTCLP

\begin{tabular}{|c|l|c|}
\hline Variable & \multicolumn{1}{|c|}{ Descripción } & Tipo \\
\hline$z_{k}$ & Valor 0 (I) si la planta de tratamiento está cerrada (no está cerrada) en el municipio $k$ & Binaria \\
\hline$y_{j t}$ & Valor 0 (I) si la instalación j está cerrada (abierta) en el periodo $t$ & Binaria \\
\hline$x_{j i t}$ & Cantidad de residuo llevado del municipio $i$ al centro de transferencia $j$ el año $t$ & Continua \\
\hline$h_{j k l t}$ & $\begin{array}{l}\text { Cantidad de residuo compactado llevado del centro de transferencia } j \text { a la planta } \\
\text { de tratamiento situada en el municipio } k\end{array}$ & Continua \\
\hline
\end{tabular}

utilizando CPLEX 10.0. En la Tabla 4 se puede observar que, si bien para pequeños problemas se llega pronto a la solución óptima, cuando los problemas tienen mayor dimensión crece el tiempo de resolución y llega un momento en el que no se pueden resolver.

\section{Modelo STPNTCLP estocástico}

El modelo de localización dinámica, planteado primero de forma determinista, se presenta ahora en su variante estocástica para distintos escenarios posibles.

En cualquier modelo estocástico existen parámetros con incertidumbre, muy usuales cuando existen decisiones de diseño cuyas implicaciones son de varios años. En este problema los parámetros con incertidumbre son: la cantidad de residuos generados en el futuro en cada municipio y el coste del combustible, que influyen en los costes de recogida y transferencia.

El problema estocástico se resuelve con un enfoque bi-etápico. En la primera fase, (con incertidumbre) se obtienen los valores de las variables de decisión de diseño («dónde» y «cuándo», dónde colocar la planta de tratamiento, dónde colocar los centros de transferencia y cuándo utilizarlos). El resto de variables (operativas) dependen para cada escenario (es decir de la segunda etapa).

Tabla 4

Tiempo de resolución y cercanía al óptimo de varios ejemplos del problema STPNTCLP

\begin{tabular}{|c|c|c|c|}
\hline Problema & Tamaño & Tiempo & Óptimo \\
\hline 1 & $88 \times 88 \times 9$ & - & - \\
2 & $40 \times 40 \times 9$ & $22,23 \mathrm{~min}$ & Sí \\
3 & $20 \times 20 \times 5$ & $74,6 \mathrm{seg}$ & Sí \\
4 & $9 \times 9 \times 3$ & $1 \mathrm{seg}$ & Sí \\
5 & $3 \times 3 \times 1$ & $<1 \mathrm{seg}$ & Sí \\
\hline
\end{tabular}

En las tres tablas siguientes se presentan los índices, parámetros y variables del modelo.

El modelo matemático se expresa de la siguiente forma:

$$
\begin{aligned}
& \min \sum_{j, t} F \cdot y_{j, t}+ \\
& +\sum_{s} p^{s}\left[\sum_{j, k, t} h_{j k t}^{s} \cdot d_{j k} \cdot c T R^{s}+\sum_{i, j, t} x_{i j t}^{s} \cdot d_{i j} \cdot c R E^{s}\right]
\end{aligned}
$$

sujeto a:

$$
\begin{aligned}
& \sum_{j} x_{i j t}^{s}=A_{i t}^{s}, \forall i, \forall t, \forall s \\
& x_{i j t}^{s} \leq y_{j t} * C A P, \quad \forall i, \forall j, \forall t, \forall s \\
& \sum_{i, j} x_{i j t}^{s} \leq \sum_{j} y_{j t} * C A P, \forall t, \forall s \\
& \sum_{k} h_{j k t}^{s}=\sum_{i} x_{i j t}^{s}, \forall j, \forall t, \forall s \\
& \sum_{j} h_{j k t}^{s} \leq z_{k} * \text { CAPplanta, } \forall k, \forall t, \forall s
\end{aligned}
$$

Tabla 5

Índices del problema STPNTCLP estocástico

\begin{tabular}{|c|l|c|}
\hline Índices & \multicolumn{1}{|c|}{ Descripción } & Rango \\
\hline$i$ & Municipios productores de residuo & I ..M \\
\hline$j$ & Posibles centros de transferencia & $1 \ldots M$ \\
\hline$k$ & $\begin{array}{l}\text { Municipios candidatos para la } \\
\text { planta de tratamiento }\end{array}$ & $1 \ldots M$ \\
\hline$t$ & Períodos & $1 \ldots T$ \\
\hline$s$ & Escenarios & $1 \ldots S$ \\
\hline
\end{tabular}


Tabla 6

Parámetros del problema STPNTCLP estocástico

\begin{tabular}{|c|l|}
\hline Parámetros & \multicolumn{1}{c|}{ Descripción } \\
\hline$A_{i t}^{s}$ & Producción de residuo de cada municipio en el año $t(\mathrm{en} T \mathrm{~m})$ \\
\hline$C A P_{j}$ & Capacidad anual de un centro de transferencia $j$ (en Tm) \\
\hline$C T R^{s}$ & $\begin{array}{l}\text { Coste medio de transferencia del residuo compactado entre un centro de transferencia y la planta de } \\
\text { tratamiento }(\text { en } € / \mathrm{km} \cdot \mathrm{Tm})\end{array}$ \\
\hline$C R E^{s}$ & $\begin{array}{l}\text { Coste medio de transporte del residuo recogido en cada municipio y llevado al centro de transferencia } \\
(\text { en } € / \mathrm{km} \cdot \mathrm{Tm})\end{array}$ \\
\hline$F_{j}$ & Costes fijos del centro de transferencia $j($ en $€)$ \\
\hline$P_{s}$ & Probabilidad del escenario $s$ \\
\hline
\end{tabular}

Tabla 7

Definición de las variables del problema STPNTCLP estocástico

\begin{tabular}{|c|l|c|c|}
\hline Variable & \multicolumn{1}{|c|}{ Descripción } & Tipo & Etapa \\
\hline$z_{k}$ & Valor 0 (I) si la planta de tratamiento está (no está) en el municipio $k$ & Binaria & Primera \\
\hline$y_{j t}$ & $\begin{array}{l}\text { Valor 0 (I) si la instalación j está cerrada (abierta) en el periodo } t \\
\text { en el escenario } s\end{array}$ & Binaria & Segunda \\
\hline$x_{j j t}^{s}$ & $\begin{array}{l}\text { Cantidad de residuo llevado del municipio } i \text { al centro de transferencia } j \\
\text { en el periodo } t \text { en el escenario } s\end{array}$ & Continua & Continua \\
\hline$h_{j k k t}^{s}$ & $\begin{array}{l}\text { Cantidad de residuo compactado llevado del centro de transferencia } j \\
\text { a la planta de tratamiento situada en el municipio } k \text { en el periodo } t\end{array}$ & \\
\hline
\end{tabular}

$\sum_{k} z_{k}=1$

Si el problema determinista es de difícil solución, el estocástico asociado es todavía más difícil ya que su complejidad es aún mayor.

\section{Enfoque algoritmos genéticos}

Debido a la dificultad de resolución de las variantes deteriminista y estocástica del problema STPNTCLP (como se ha comentado en los apartados anteriores), y también a que, generalmente, para problemas reales con incertidumbre hablar de óptimo es un poco arriesgado y que es aceptable llegar a soluciones buenas, se ha planteado la resolución del problema utilizando algoritmos genéticos.

Este enfoque mediante algoritmos genéticos, que se puede utilizar tanto para el problema determinista como para el estocástico del STPNTCLP, se presenta en esta comunicación aplicado al determinista (más sencillo). A su vez este mismo enfoque deriva en dos variantes. Ambas se presentan a continuación.

\section{I. Variante I del enfoque mediante AG. Función de supervivencia calculada con un modelo LP}

En este enfoque basado en los algoritmos genéticos se van a presentar sólo aquellos aspectos específicos y más relevantes para este problema concreto (individuos, cálculo de la función de supervivencia, cruces, mutaciones, soluciones no factibles). El resto de aspectos son análogos a los que aparecen en cualquier enfoque basado en algoritmos genéticos.

\subsubsection{Individuo}

Un individuo está compuesto de dos cromosomas (ver Figura 3). El primer cromosoma (la matriz binaria) expresa si un centro de transferencia que esté en el municipio $i$ ( fila i) se abre ( 1 ) o no (0) en el periodo $t$ (columna $t$ ). El segundo cromosoma es un número entre ( $\mathrm{l}$ y $\mathrm{N}$ ) que indica en qué municipio está la planta de tratamiento (en la figura el número 23). Como se puede observar, el individuo contiene toda la información relativa a las variables de decisión de diseño (dónde ubicar las instalaciones y cuándo abrirlas). 
Figura 3

Individuo (izda.) y método de cálculo de la función de supervivencia

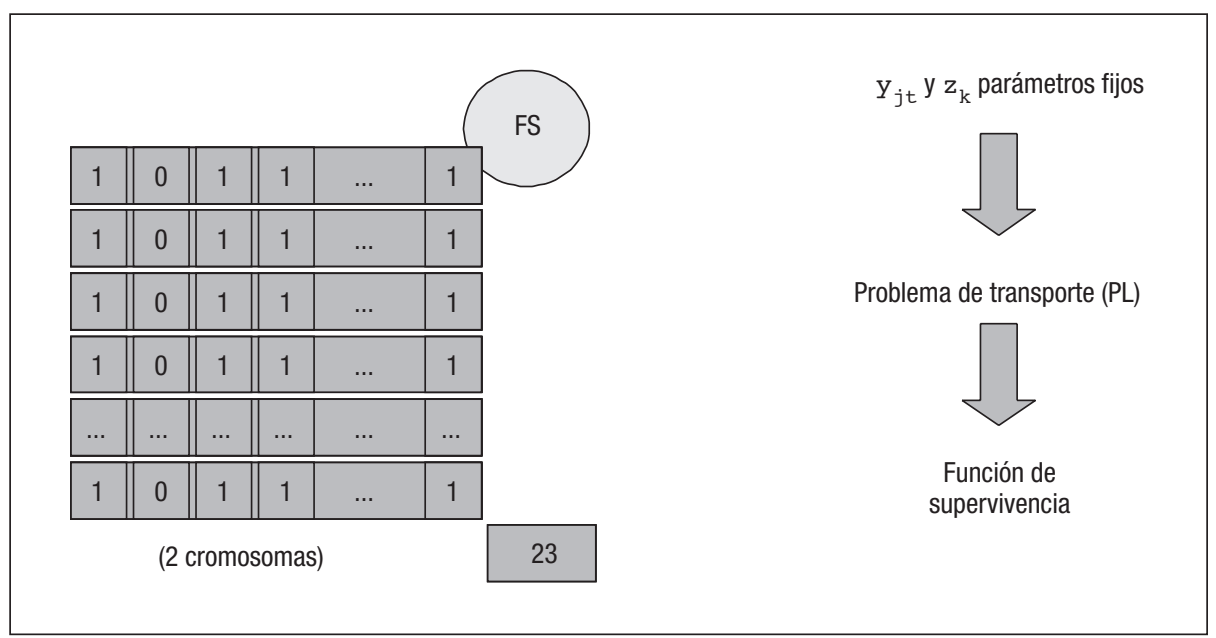

\subsubsection{Cálculo de la función de supervivencia}

Con la información de un individuo es sencillo calcular el coste asociado a dicha solución. Habría que resolver el modelo STPNTCLP representado en las ecuaciones $[1-7]$, pero teniendo en cuenta que las variables $y_{j t} y z_{k}$ ahora son parámetros de un modelo que ya no tiene variables binarias (LP). Este modelo es de fácil y rápida resolución.

Una vez obtenido el coste de la solución es sencillo calcular la función de supervivencia haciendo el inverso del coste

\subsubsection{Cruces}

Se han definido tres tipos de cruces, en función de la información que intercambian las soluciones:

I. Intercambio de la información relativa a los periodos (columnas). Aleatoriamente se hace un

Figura 4

Cruce de dos individuos compartiendo información relativa a los periodos

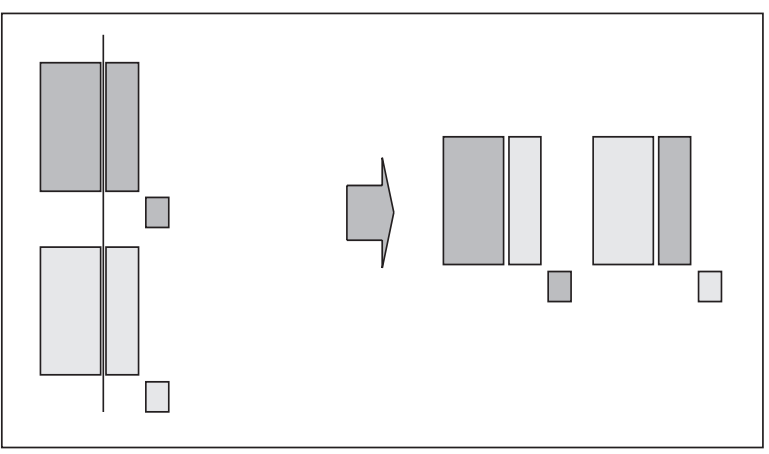

corte en las matrices de ceros y unos (primer cromosoma) y se construyen los individuos hijos a partir del segundo cromosoma y la primera parte del primer cromosoma de un padre junto con la información correspondiente a la otra mitad del primer cromosoma del otro padre. De forma análoga se construye el otro hijo.

Figura 5

Cruce de dos individuos compartiendo información relativa a los centros de transferencia

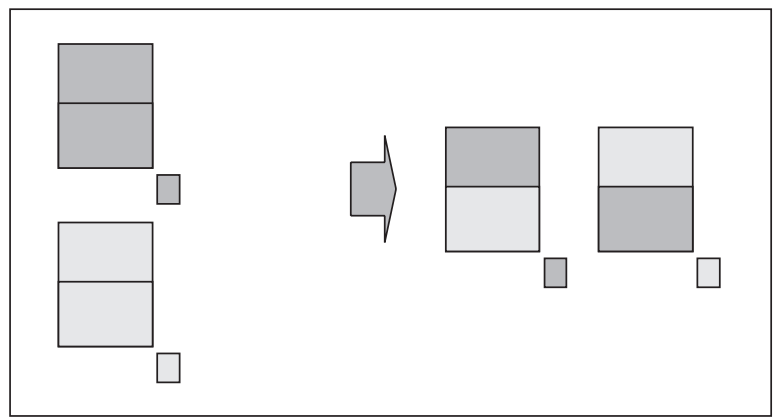

Figura 6

Cruce de dos individuos compartiendo información relativa a la localización de la planta

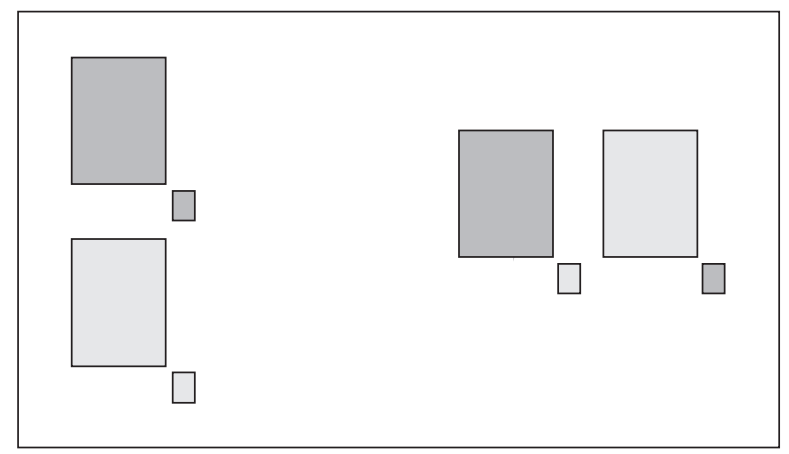


2. Intercambio de la información relativa a los centros de transferencia. Ahora, análogamente al cruce anterior, el corte de las matrices se hace horizontal provocando un intercambio de la información relativa al uso de los centros de transferencia (filas).

3. Intercambio de la información relativa a la localización de la planta de tratamiento. En este caso, los padres cruzan el segundo cromosoma, sin tocar el primero.

Estos tres cruces son independientes, y sólo se puede realizar un cruce en el mismo momento. Aleatoriamente en cada instante se decide qué tipo de cruce realizar.

\subsubsection{Mutaciones}

Las mutaciones se producen cambiando un cero por un uno (o viceversa) en el primer cromosoma; o aleatoriamente cambiando el valor del segundo cromosoma.

\subsubsection{Soluciones no factibles}

Al realizar los cruces entre individuos y las mutaciones, que se realizan de forma aleatoria, se pueden generar soluciones no factibles. Para convertir estos nuevos individuos en factibles se tiene que implementar un procedimiento que «arreg/e»la infactibilidad abriendo centros de transferencia (cambiando ceros por unos en el primer cromosoma).

\subsection{Variante 2 del enfoque mediante AG. Función de supervivencia calculada con un modelo MIP}

Se plantea una variante al enfoque presentado en el apartado anterior. Las diferencias son las siguientes:

- Los individuos están formados sólo por un único cromosoma, el relativo a los centros de transferencia (matriz binaria).

- La función de supervivencia se calcula resolviendo un modelo asociado que en este caso es un modelo MIP en el que existen variables $K$ variables binarias $\left(z_{k}\right)$.

En esta variante se busca aprovechar las ventajas relativas a la existencia de un único cromosoma y la simplificación del algoritmo, a consta de las desventajas relativas a la complicación del cálculo de la función de supervivencia (ahora un modelo MIP, aunque de «pocas» variables binarias).

\section{Conclusiones}

En este artículo se ha presentado un problema de localización de las instalaciones de transferencia y tratamiento en un sistema de recogida de residuos. Junto al problema también se han presentado los modelo MIP deterministas y estocásticos correspondientes (STPNTCLP) que ayuda a la toma de decisiones en el problema anterior.

Además se ha planteado un enfoque basado en algoritmos genéticos que intenta resolver los mode-

Figura 7

Variante 2 del enfoque AG (individuo y procedimiento de cálculo de la función de supervivencia)

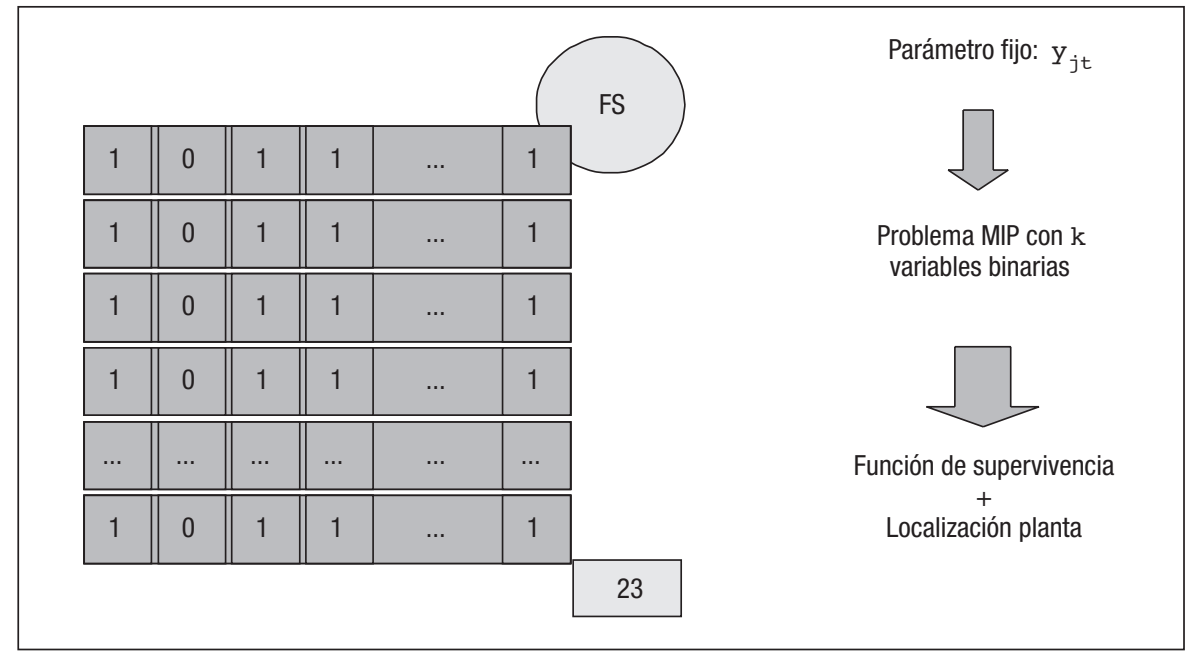


los anteriores dada la dificultad de la resolución de forma exacta.

\section{Referencias}

ANTUNES, A., y PEETERS, D. (2000). A dynamic optimization model for school network planning. SocioEconomic Planning Sciences, vol. 34, pp. I0 I - 120.

BOOKER, L. B.; GOLDBERG, D. E., y HOLLAND, J. H. (1989). Classifier systems and genetic algorithms. Artificial Intelligence, vol 40, Issues I-3, pp. 235-282.

BOSE, I.; ERYARSOY, E., y HE, L., (2003). Multi-period design of survivable wireless access networks under capacity constraints. Decision Support Systems.
HINOJOSA, Y.; PUERTO, J., y FERNANDEZ, F. R., (2000). A multiperiod two-echelon multicommodity capacitated plant location problem. European Journal of Operational Research, vol. 123, pp. 27|-29|.

FLEISCHMANN, M., et al. (200I). The impact of product recovery on logistics network design. Production and Operations Management, vol I0, pp. I56- 173.

SALDANHA, F., y CAPTIVO, M. E. ( 1 998). A heuristic approach for the discrete dynamic location problem. Location Science, vol. 6, pp. 2 I I-223.

GAREY, M. R., y JOHNSON, D. S. ( 1979). Computers and Intractability: A Guide to the Theory of NPCompleteness. W.H. Freeman and Company. 